\title{
Controlled Feature Adjustment for Image Processing and Synthesis
}

\author{
Eduardo Martínez-Enríquez \\ Instituto de Óptica, CSIC \\ Madrid, Spain \\ eduardo.martinez@io.cfmac.csic.es
}

\author{
Javier Portilla \\ Instituto de Óptica, CSIC \\ Madrid, Spain \\ javier.portilla@csic.es
}

\begin{abstract}
Feature adjustment, understood as the process aimed at modifying at will global features of given signals, has cardinal importance for several signal processing applications, such as enhancement, restoration, style transfer, and synthesis. Despite of this, it has not yet been approached from a general, theory-grounded, perspective. This work proposes a new conceptual and practical methodology that we term Controlled Feature Adjustment (CFA). CFA provides methods for, given a set of parametric global features (scalar functions of discrete signals), (1) constructing a related set of deterministically decoupled features, and (2) adjusting these new features in a controlled way, i.e., each one independently of the others. We illustrate the application of CFA by devising a spectrally-based hierarchically decoupled feature set and applying it to obtain different types of image synthesis that are not achievable using traditional (coupled) feature sets.

Index Terms-decoupled features; nested normalizations; controlled feature adjustment; no-reference synthesis; meta-synthesis
\end{abstract}

\section{INTRODUCTION}

Feature adjustment (FA) can be thought of as the process of applying global transformations to signals aimed at modifying some of their features. It has cardinal importance for a number of signal processing applications, such as enhancement [1], restoration [2]-[5], style transfer [6], [7] or synthesis (noreference [8], [9] or example-based [10]-[13]). Despite such a central role, to the best of our knowledge FA has not yet been approached from a general, comprehensive and theorygrounded perspective. In this paper we focus on parametric $\mathrm{FA}^{1}$. In that case, we have a set of features defined as mathematical functions (typically global, real, and shift invariant, e.g., sample statistics) of discretized signals, and the goal is to modify the signal to match some desired values at the output of these functions.

As shown in Table 1, there are several possibilities of FA, depending on the origin of the desired feature values we want to impose and on the type of image (seed) on which we impose those values. In case the features' values come from having previously analyzed another image, then we do feature/style transfer or example-based synthesis (for features imposed onto

\footnotetext{
Funded by the Spanish Government grant FIS2016-75891-P.

${ }^{1}$ From now on we will refer to parametric FA simply as FA. Likewise, from now on we will consider that the involved signals are digital images, although all methods explained here are equally applicable to other types of signals.
}

TABLE I

SOME APPLICATIONS OF FEATURE ADJUSTMENT

\begin{tabular}{|c|c|c|}
\hline Seed $\rightarrow$ & Observed & Synthetic \\
\hline Feature Origin $\downarrow$ & & Data \\
\hline $\begin{array}{l}\text { Analyzed } \\
\text { Image }\end{array}$ & $\begin{array}{l}\text { Feature } \\
\text { Transfer }\end{array}$ & $\begin{array}{c}\text { Example-Based } \\
\text { Synthesis }\end{array}$ \\
\hline $\begin{array}{l}\text { Estimated } \\
\text { from Observ. }\end{array}$ & Restoration & $\begin{array}{c}\text { "Smart } \\
\text { Synthesis" }\end{array}$ \\
\hline Arbitrary & $\begin{array}{c}\text { Enhancement } \\
\text { \& Effects }\end{array}$ & $\begin{array}{c}\text { No-Reference } \\
\text { Synthesis }\end{array}$ \\
\hline Combined & $\begin{array}{l}\text { Combination } \\
\text { Morphing }\end{array}$ & $\begin{array}{c}\text { Mixing } \\
\text { Synthesis }\end{array}$ \\
\hline $\begin{array}{l}\text { Sampled } \\
\text { (random) }\end{array}$ & $\begin{array}{c}\text { "Random } \\
\text { Perturbation" }\end{array}$ & $\begin{array}{c}\text { Meta- } \\
\text { Synthesis }\end{array}$ \\
\hline
\end{tabular}

another observed image or on synthetic data e.g., a computer generated pseudo-random image, respectively). In the case of the features' values not coming from analyzing another image, but having arbitrary values in their allowed ranges, then we do image enhancement or no-reference synthesis (for seeds coming from observed images or synthetic pseudorandom seeds respectively). Other interesting possibilities are to combine (e.g., average) feature vectors, leading to morphing or mixing-synthesis (on observed images or synthetic data respectively), or to sample the feature vectors from a statistical model, leading to random-perturbation or meta-synthesis (on observed images or synthetic data respectively).

Note that all shaded applications in Table 1 (the restoration case is special, as it lies in between both groups) require the features to be independently modifiable. This is not the case with usual feature sets, which suffer from deterministic coupling, giving rise to algebraically incompatible combinations (e.g., high skewness and low kurtosis [14], [15]). Two difficulties related to feature coupling are that we don't know (1) how to check if their values are mutually compatible (compatibility assesment), and (2) how to obtain sets of compatible values, apart from obtaining them from analyzing other images 
(compatibility enforcement). Thus, it is not surprising that applications such as style transfer and example-based synthesis have flourished, whereas others based on imposing arbitrary feature values have not.

In this paper we propose a controlled featured adjustment (CFA) method that overcomes the feature coupling problem, thus allowing to modify the value of each feature independently of the others. The method is based on our recent advances in deterministic feature decoupling [15], [16]. Specifically, we introduce here the nested normalizations (NNs) method for synthesis, as a counterpart of its version for analysis [15], [16]. NNs method allows to build a new set of decoupled features from a given coupled set. When applied to analyzing an image, NNs hierarchically normalizes the image and measures the values of its decoupled features. In synthesis, NNs performs CFA, by imposing some desired values of decoupled features (measured previously or generated in different ways) onto a target image. We also introduce here a decoupled feature set based on differences of variance between wavelet subbands (we termed it the DifVar set), and use it to illustrate mixing synthesis, no-reference random synthesis, and meta-synthesis examples. These advanced synthesis techniques are not possible using traditional (coupled) feature sets. The rest of the paper is organized as follows. Section II motivates the proposed controlled featured adjustment, summarizes our previous work on the nested normalization method for analysis and introduces the new nested normalization approach for synthesis. Section III presents our novel decoupled DifVar set. Experimental results are provided in Section IV. Section V concludes the paper.

\section{FROM UNCONTROLLED TO CONTROLLED FEATURE ADJUSTMENT (CFA)}

The two basic properties that a CFA method requires, to provide the necessary control, are reversibility and orthogonality. Reversibility of the adjustment provides stability to the FA, by ensuring there is no information loss. Orthogonality of the gradients allows for independently adjusting each feature's value without affecting the others.

\section{A. Reversibility: Integrating the gradient (instead of project- ing)}

Assume we have an image $\vec{x}_{0} \in R^{N}$ and a given feature $f(\vec{x}) \in R$, a scalar differentiable function of our image $\vec{x}_{0}$. We term feature adjustment any transformation $T: R^{N} \rightarrow R^{N}$, $T\left(\vec{x} ; f, f_{d}\right)$ aimed at adjusting $f(T(\vec{x}))$ to a certain desired value $f_{d}: f\left(T\left(\vec{x}_{0} ; f, f_{d}\right)\right)=f_{d}$. A basic requirement on $T$ is that it should adjust $f$ by changing $\vec{x}_{0}$ as little as possible. If we use the Euclidean metric then $T$ is the (shortest, if more than one) orthogonal projection of $\vec{x}_{0}$ onto the manifold $f^{-1}\left(f_{d}\right)$. There are many examples of feature adjustment procedures based on orthogonal projections (e.g. [2]-[5]). In differential terms, the change direction that produces the fastest change in $f(\vec{x})$ is its gradient, $\nabla f(\vec{x})$. A usually simpler alternative to compute the orthogonal projection consists in following the feature's gradient direction at $\vec{x}_{0}$ until reaching the

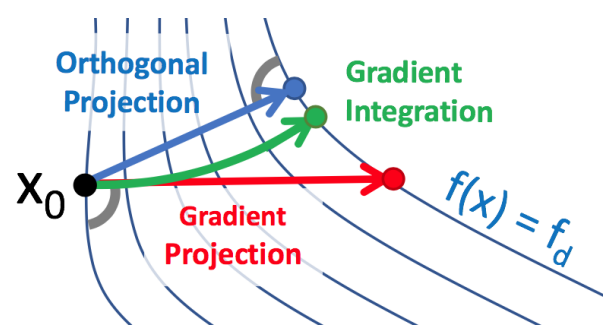

Fig. 1. An illustration of feature adjustment by projection (orthogonal \& gradient direction) vs. gradient integration.

desired value: $T\left(\vec{x}_{0} ; f, f_{d}\right)=\vec{x}_{0}+\left.\lambda\left(\vec{x}_{0} ; f, f_{d}\right) \nabla f(\vec{x})\right|_{\vec{x}=\vec{x}_{0}}$, with $\lambda\left(\vec{x}_{0} ; f, f_{d}\right)$ the smallest in absolute value imposing $f\left(\vec{x}_{0}+\left.\lambda \nabla f(\vec{x})\right|_{\vec{x}=\vec{x}_{0}}\right)=f_{d}$. This method was applied in successive iterations in [13] for texture synthesis. However, the two above methods have a serious problem: they are not reversible, i.e., they do not accomplish

$$
T\left(T\left(\vec{x}_{0} ; f, f_{d}\right) ; f, f\left(\vec{x}_{0}\right)\right)=\vec{x}_{0} .
$$

Lack of reversibility ${ }^{2}$ breaks symmetry and destroys information. It is related to the signal's trajectory drift when the desired feature value is adjusted in several steps, instead of in one step. The solution to the problems above is to follow the gradient direction everywhere, differentially, in a curved trajectory, instead of projecting. This corresponds to solving the ordinary differential equation (ODE):

$$
\frac{d \vec{x}(t)}{d t}=\nabla f(\vec{x}(t))
$$

with $\vec{x}(0)=\vec{x}_{0}$, and finding the smallest (in absolute value) $t_{0}=\arg _{t}\left\{f(\vec{x}(t))=f_{d}\right\}$. Now we have that $T\left(\vec{x}_{0} ; f ; f_{d}\right)=\vec{x}\left(t_{0}\right)=F\left(\vec{x}, t_{0} ; \vec{x}_{0}\right)$, being $F\left(\vec{x}, t ; \vec{x}_{0}\right)$ the ODE solution, which, being an integral function, fulfils: $F\left(\vec{x}, t_{2} ; F\left(\vec{x}, t_{1} ; \vec{x}_{0}\right)\right)=F\left(\vec{x}, t_{1}+t_{2} ; \vec{x}_{0}\right)$. This ensures reversibility (by choosing $t_{2}=-t_{1}$ ) and independence of the intermediate steps $\left(F\left(\vec{x}, t_{3} ; \vec{x}_{0}\right)\right.$, with $t_{3}=t_{1}+t_{2}$, is independent on the particular values of $t_{1}, t_{2}$, whenever they add to $t_{3}$. ${ }^{3}$. Figure 1 illustrates the described forms of feature adjustment. Usually, instead of adjusting a single feature, we want to adjust a whole vector of features, $\vec{f}(\vec{x}) \in M$ (usually $M<<N$ ). In previous works of texture synthesis (e.g. [11], [13]) individual projections were sequentially iterated through all features, for many cycles, until (practical) convergence. Besides the solution being iterative, major limitations of these methods came from using deterministically coupled features, which prevented to independently modify each one in a known, fixed, range.

\section{B. Gradient orthogonality: Nested Normalizations (Analysis)}

In [16] we generalized and formalized the NNs method proposed initially in [15]. As demonstrated in [16], given a set of sequentially ordered differentiable features, the method provides a new related set having mutually orthogonal gradients for all the input space (i.e., a set of decoupled features).

\footnotetext{
${ }^{2}$ Though orthogonal and gradient projections are the reverse of each other ${ }^{3}$ Reversibility it's only broken when reaching a feature extreme.
} 


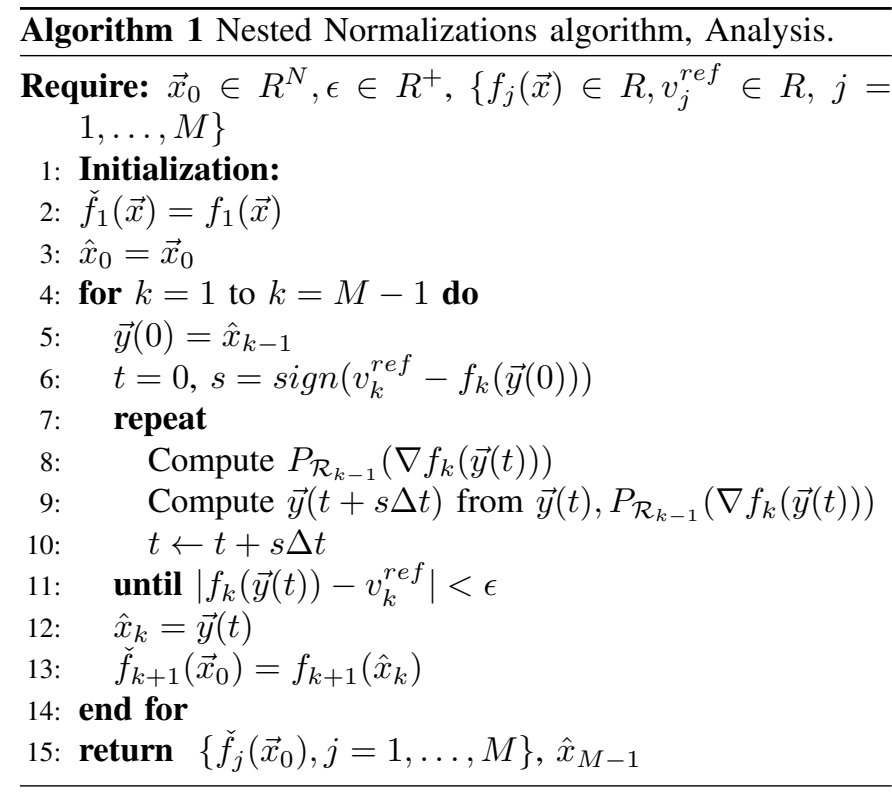

The new decoupled features are obtained through feature translation [16] of the input vector to nested reference manifolds providing normalized values for the features. Although we refer the reader to [16] for the mathematical details, here we give a summary of the NNs algorithm. Algorithm 1 describes NNs analysis method: the sequential normalization of the features of a given sample $\vec{x}_{0}$. The decoupled features are the original $k$-th feature evaluated on the $(k-1)$-normalized sample $\hat{x}_{k}$ : $\check{f}_{k}\left(\vec{x}_{0}\right)=f_{k}\left(\hat{x}_{k-1}\right)$. The method imposes sequentially a new reference value to an added feature, while not changing the previous features. This is achieved by translating $\hat{x}_{k-1}$ along the gradient of the $k$-th feature being adjusted, but with the gradient projected onto the orthogonal complement of the previous features' gradients. Thus, the vector's trajectory does not leave its reference manifold $\mathcal{R}_{k-1}$. The integration continues until reaching the next reference manifold $\mathcal{R}_{k}$, where the next decoupled feature is obtained, and the process continues until all $M$ decoupled features are computed. Note also that the reference manifolds fulfil $\mathcal{R}_{k}=\vec{f}_{k}^{-1}\left(\vec{v}_{k}^{r e f}\right), k=1, \ldots, M$, with $\vec{f}_{k}(\vec{x})=\left[f_{j}(\vec{x})\right], \vec{v}_{k}^{r e f}=\left[v_{j}^{r e f}\right], j=1, \ldots, k$, and that they are nested: $\mathcal{R}_{M} \subset \mathcal{R}_{M-1} \subset \cdots \subset \mathcal{R}_{1} \subset \mathcal{R}_{0}=R^{N}$. Figure 2 describes the method graphically.

\section{CFA: Nested Normalizations (Synthesis)}

The previous method must be completed with its synthesis counterpart when our aim is to impose a set of desired values $\left\{v_{j}^{\text {des }}, j=1, \ldots, M\right\}$ on a given signal sample. The synthesis process performs feature translations in the reverse order as in the analysis, starting from an $M-1$-normalized seed $\hat{w}_{M-1}$ and then sequentially imposing the desired decoupled feature values $f_{k}\left(\hat{z}_{k-1}\right)=\check{f}_{k}\left(\vec{z}_{f}\right)=v_{k}^{\text {des }}$, for $k=M, \ldots, 1$, without changing the reference values for $j<k$. Thanks to the reversibility of the feature translations, the so obtained intermediate sample vectors are normalized versions of the final result $\vec{z}_{f}$. This implies that $\vec{z}_{f}$ returns the desired de-
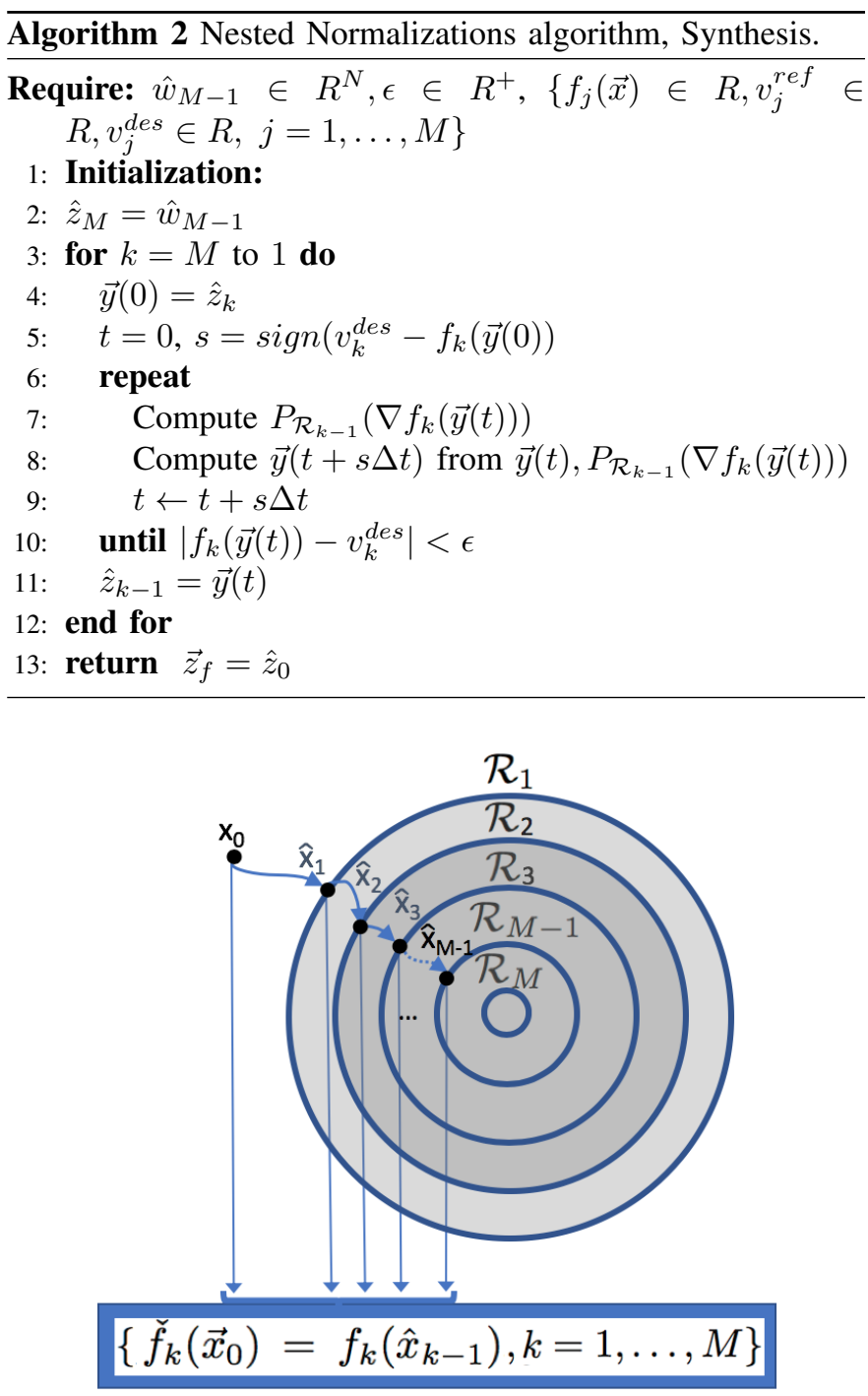

Fig. 2. Nested Normalizations: Analysis.

coupled feature values when analyzed (consistency condition of the synthesis w.r.t. the analysis). Algorithm 2 and Fig. 3 summarize the synthesis algorithm.

\section{DIFVAR: A DECOUPLED FEATURE SET}

Using the variance at the output of overcomplete representations such as $\mathrm{x}$-let (wavelet-like) subbands is a common way to obtain parsimonious spectral descriptions (see, e.g. [10]). However, due to the subbands' spectral overlapping, these features are heavily coupled. Thus, the associated adjustment problem has been traditionally addressed by iterating (see, e.g. [11], [13]). However, this does not solve the feature coupling problem, i.e., some subbands variance combinations remain incompatible. Here we propose a parsimonious wavelet-based spectral feature scheme providing decoupled features. It is based on the steerable pyramid [17], with just two orientations. Figure 4 shows a scheme of the proposed feature set. Each extracted feature has been numbered, and a 


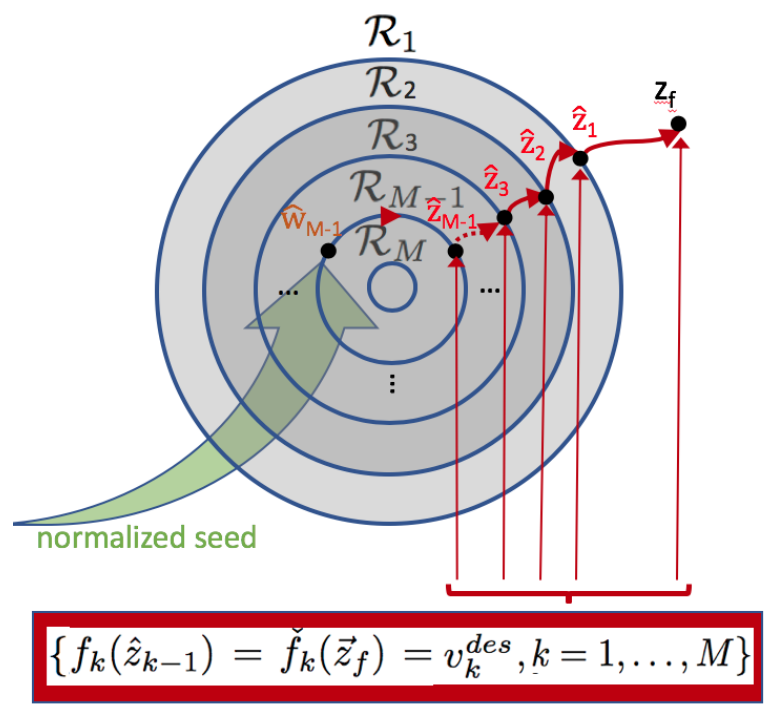

Fig. 3. Nested Normalizations: Synthesis.

curved line has been depicted marking the sequential path of normalizations. It aims to illustrate that, following the NNs algorithm, each feature is measured sequentially after having adjusted the previous one to its reference (normalized) value (same, in reversed order, during the synthesis). It uses, besides the pixels' mean and variance, the differences of variance at the output of adjacent bands, through radial frequency and orientation. The associated ODEs for integrating the gradients are easily obtained in the Fourier domain (expressions are omitted here for brevity), as the gradient of the sample variance at the output of a filter is proportional to the output of the filter (the latter squared in modulus). As a consequence, the associated ODE solutions for the involved feature translations are simple exponentials. What is essential when building a hierarchical order sequence for the features is to avoid introducing arbitrary asymmetries between them. For the reference values, required for the nested normalizations, we have chosen the expected values for a white zero-mean univariate white noise. Compared to successful image models, as the ones used for textures, the DifVar decoupled feature set is little more than a toy example, clearly not intended to provide state-of-the-art image quality results. However, it serves to illustrate the potentials of our CFA scheme, as it allows to carry out some tasks that can't be done using traditional (coupled) parametric features.

\section{CFA EXPERIMENTS}

\section{A. Decoupling effect of DifVar}

Figure 5 shows two scatter plots corresponding to the difference-of-variance features 3rd and 5th (see Fig. 4), measured on 30, 000 standardized 8x8 Gaussian noise images. On the top, in blue, using the conventional, non-decoupled way, directly measuring differences of adjacent subband variance values in the wavelet domain. On the bottom, in red, obtained through NNs in the DifVar scheme. We see how deterministic

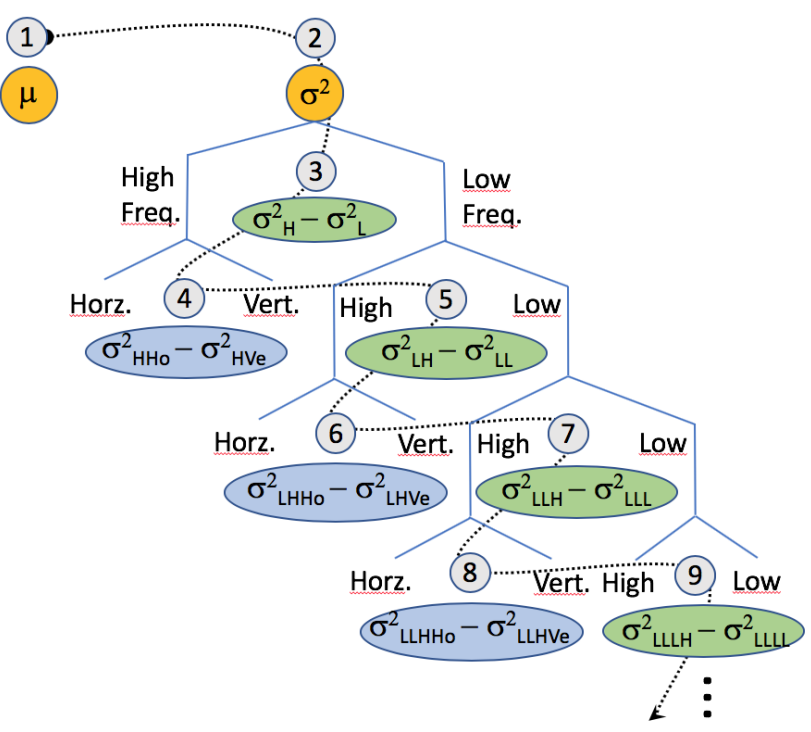

Fig. 4. DifVar, a hierarchically decoupled feature set providing parsimonious description of the image spectrum.

coupling in the coventional difference-of-variance scheme generates non-separable joint output domains for the feature vectors (causing an oblique boundary on the top). In contrast, decoupled features have joint separable domains, i.e., each decoupled feature has its own fixed range of possible values independent of the values of the other features. This greatly facilitates, among other possibilities (1) modeling and sampling of the joint feature pdf (meta-synthesis); (2) doing arbitrary, no-reference, synthesis or enhancement; and (3) mixing arbitrary feature vectors, all of them without risking to end up in forbidden (out of the joint output domain) feature values combinations. Note that this risk quickly grows when increasing the dimensionality of the (coupled) feature vector, potentially giving rise to unmanageable complex joint output domains.

\section{B. Synthesis examples using DifVar}

Here we show some possibilities of CFA to successfully achieve different FA examples presented on Table I. We used some of the natural textured images from Brodatz album [18], and our DifVar set with $M=15$ features.

1) Mixing synthesis: We synthesized a sequence of images by mixing the parameters of two real textures in different proportions. First, we obtained the feature vectors of the two textures using the NNs analysis Algorithm 1. Then, we synthesized the different images of the sequence using a common random seed and imposing an intermediate feature vector, by CFA using Algorithm 2. The feature vectors were obtained, for each image, as the weighted average between the feature vectors of the original textures. Figure 6 shows 9 images synthesized using a vector of weights $\mathbf{w}_{\mathbf{1}}=\left[\begin{array}{lllllllll}0 & 1 / 8 & 2 / 8 & 3 / 8 & 4 / 8 & 5 / 8 & 6 / 8 & 7 / 8 & 1\end{array}\right]$ for the feature vector of the first real texture and $\mathbf{w}_{\mathbf{2}}=1-\mathbf{w}_{\mathbf{1}}$ for 

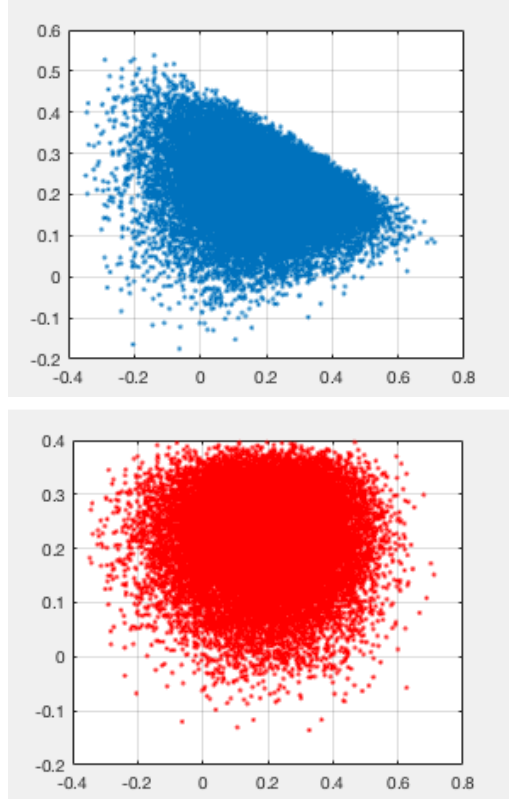

Fig. 5. Scatter plots of the 3rd and 5th DifVar features, showing the decoupling effect of Nested Normalizations: top, conventional (coupled) version of DifVar features; bottom, real DifVar (decoupled) features.

the second texture. The result (improving previous attempts, e.g., [13]) is a smooth progression of homogeneous texture samples.

2) No-reference synthesis: We generated a mosaic of noreference synthetic images, as shown in Fig. 7, by choosing a selection of textures obtained by sampling uniformly the valid (fixed) ranges of the features values. This gives a rough idea of the visual expressiveness of the used feature model.

3) Meta-synthesis: Finally, we attacked the problem of meta-synthesis ${ }^{4}$, i.e., modeling and reproducing the statistical fluctuations affecting samples from a given texture. Metasynthesis was done in four steps: (1) obtain the feature values for a set of samples from the same texture (here we used patches from a big image, but a set of samples from a nonergodic random field could be used as well); (2) build a statistical model to characterize the observed distribution of feature values (here a multivariate Gaussian); (3) sample from that model a set of feature values; and (4) sample the texture patches, subject to the previously sampled features. Note that this can not be done using non-decoupled features, as there are no warranties of the sampled feature values to be mutually compatible. CFA allowed to successfully attack this problem, as shown in Fig. 8.

\section{CONCLUSIONS}

We have presented a new conceptual and algorithmic framework (termed Controlled Feature Adjustment) for signal

\footnotetext{
${ }^{4}$ Binary texture meta-synthesis was made in [9]. However, that method did not provide any warranty of keeping the combined parameters consistent, and, thus, an ad-hoc pruning of the pdf was necessary.
}
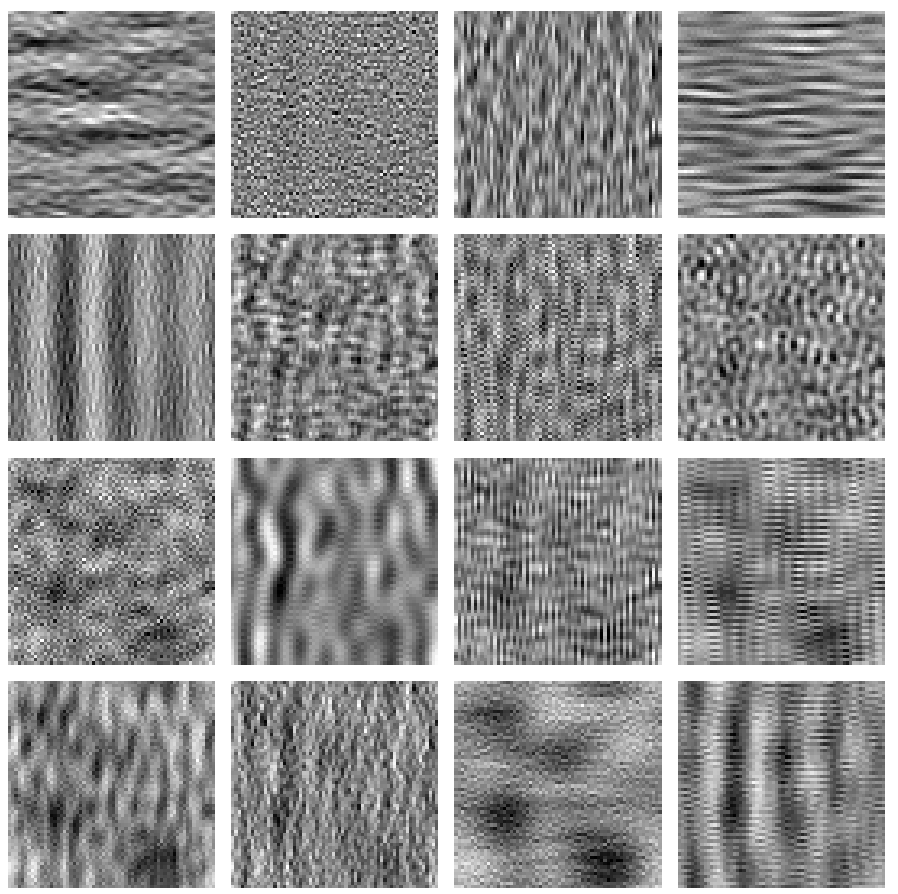

Fig. 7. No-reference random synthesis using CFA on DifVar.

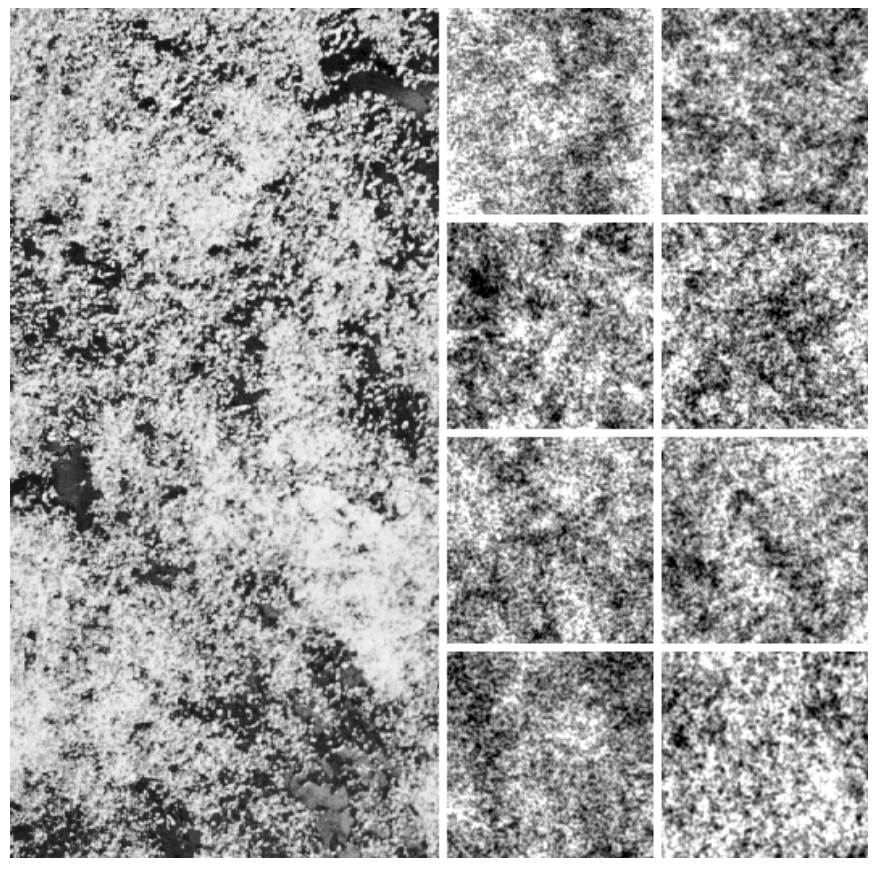

Fig. 8. Meta-synthesis: features of samples from a texture (left) are modelled as a pdf (multivariate Gaussian), and meta-sampled - first in the feature and then in the image space (right). 

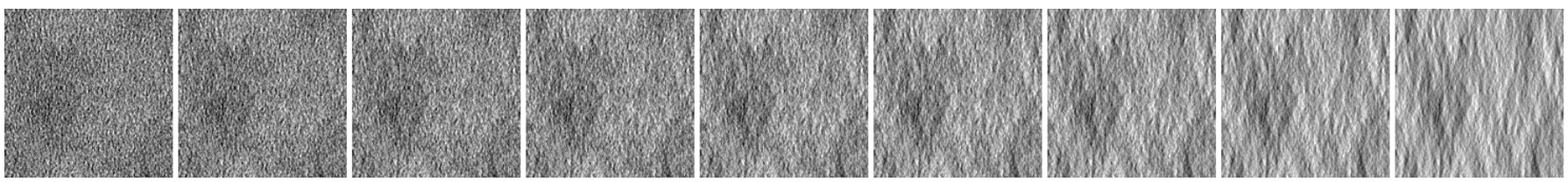

Fig. 6. Texture mixing example: CFA allows to navigate along an arbitrary curve in the feature space (here a straight line). The feature vectors were obtained, for each image, as the weighted average between the feature vectors of the original textures. See text for details.

processing and synthesis. It is based on measuring and adjusting algebraically decoupled features, by using the Nested Normalizations algorithm, both in its original analysis form, and in its new, synthesis, reversed counterpart. This allows to transform any image by freely navigating its feature space. We have built a simple example of decoupled feature set by using variance differences in wavelet subbands, and applied it to feature mixing, no-reference synthesis, and meta-synthesis. Next challenge is to build more advanced signal models (e.g., for textures or sounds) using decoupled features. This, we believe, could have, amongst others, a relevant impact on the field of perception and neural science, where being able to generate and modulate at will a wide variety of stimuli (like visual texture) in a fully controlled way is essential (see, e.g., [19]-[21]).

\section{REFERENCES}

[1] R. Hummel, "Image enhancement by histogram transformation," Computer Graphics and Image Processing, vol. 6, no. 2, pp. $184-195$, 1977.

[2] Aharon Levi and Henry Stark, "Image restoration by the method of generalized projections with application to restoration from magnitude," J. Opt. Soc. Am. A, vol. 1, no. 9, pp. 932-943, Sep 1984.

[3] M. K. Ozkan, A. M. Tekalp, and M. I. Sezan, "Pocs-based restoration of space-varying blurred images," IEEE Transactions on Image Processing, vol. 3, no. 4, pp. 450-454, July 1994.

[4] S. S. Panda, M. S. R. S Prasad, and G. Jena, "Pocs based super-resolution image reconstruction using an adaptive regularization parameter," 2011.

[5] J. Portilla and E. P. Simoncelli, "Image denoising via adjustment of wavelet coefficient magnitude correlation," in Proceedings 2000 International Conference on Image Processing (Cat. No.00CH37101), Sep. 2000, vol. 3, pp. 277-280 vol.3.

[6] J. Yosinski, J. Clune, Y. Bengio, and H. Lipson, "How transferable are features in deep neural networks?," in Advances in Neural Information Processing Systems 27, Z. Ghahramani, M. Welling, C. Cortes, N. D. Lawrence, and K. Q. Weinberger, Eds., pp. 3320-3328. Curran Associates, Inc., 2014.

[7] L. A. Gatys, A. S. Ecker, and M. Bethge, "Image style transfer using convolutional neural networks," in 2016 IEEE Conference on Computer Vision and Pattern Recognition (CVPR), June 2016, pp. 2414-2423.

[8] G. R. Cross and A. K. Jain, "Markov random field texture models," IEEE Transactions on Pattern Analysis and Machine Intelligence, vol. PAMI-5, no. 1, pp. 25-39, 1983.

[9] J. Portilla, "Quantum-computation-inspired reverse analysis texture synthesis," in 2015 IEEE International Conference on Image Processing (ICIP), Sep. 2015, pp. 596-600.

[10] J. Portilla, R. Navarro, O. Nestares, and A. Tabernero, "Texture synthesis-by-analysis based on a multiscale early-vision model," Optical Engineering, vol. 35, no. 8, pp. 2403-2417, 1996.

[11] D. J. Heeger and J. R. Bergen, "Pyramid-based texture analysis/synthesis," in Proceedings., International Conference on Image Processing, Oct 1995, vol. 3, pp. 648-651 vol.3.

[12] S. C. Zhu, Y. N. Wu, and D. Mumford, "Filters, random fields and maximum entropy (FRAME): Towards a unified theory for texture modeling," International Journal of Computer Vision, vol. 27, no. 2, pp. 107-126, Mar 1998.
[13] J. Portilla and E.P. Simoncelli, "A parametric texture model based on joint statistics of complex wavelet coefficients," International Journal of Computer Vision, vol. 40(1), pp. 49-71, 2000, ®Matlab code publicly available at http://www.cns.nyu.edu/ lcv/texture/.

[14] R. Sharma and R. Bhandari, "Skewness, kurtosis and Newton's inequality," ArXiv e-prints, Sept. 2013.

[15] J. Portilla and E. Martinez-Enriquez, "Nested normalizations for decoupling global features," in 2018 25th IEEE International Conference on Image Processing (ICIP), Oct 2018, pp. 2112-2116.

[16] E. Martinez-Enriquez and J. Portilla, "Deterministic feature decoupling by surfing invariance manifolds," in ICASSP 2020 - 2020 IEEE International Conference on Acoustics, Speech and Signal Processing (ICASSP), 2020, pp. 6049-6053.

[17] E. P. Simoncelli and W. T. Freeman, "The steerable pyramid: a flexible architecture for multi-scale derivative computation," in Proceedings., International Conference on Image Processing, Oct 1995, vol. 3, pp. 444-447 vol.3.

[18] P. Brodatz, Textures: a photographic album for artists and designers, Dover pictorial archives. Dover Publications, 1966, Available at https://multibandtexture. recherche.usherbrooke.ca/original_brodatz.html.

[19] J. Balas, "Texture synthesis and perception: Using computational models to study texture representations in the human visual system," Vision Research, vol. 46, no. 3, pp. 299 - 309, 2006.

[20] Gouki Okazawa, Satohiro Tajima, and Hidehiko Komatsu, "Image statistics underlying natural texture selectivity of neurons in macaque v4," Proceedings of the National Academy of Sciences, vol. 112, no. 4, pp. E351-E360, 2015.

[21] Jeremy Freeman, Corey M Ziemba, David J Heeger, Eero P Simoncelli, and J Anthony Movshon, "A functional and perceptual signature of the second visual area in primates," Nature Neuroscience, vol. 16, 052013. 